\title{
"Dealing with the changeable and blurry edges of living things: a modified version of property-cluster kinds"
}

\author{
María J. Ferreira Ruiz ${ }^{1,2}$ (D) Jon Umerez ${ }^{3}$
}

Received: 12 October 2017 / Accepted: 18 June 2018 /Published online: 29 June 2018

(C) The Author(s) 2018, corrected publication August $/ 2018$

\begin{abstract}
Despite many attempts to achieve an adequate definition of living systems by means of a set of necessary and sufficient conditions, the opinion that such an enterprise is inexorably destined to fail is increasingly gaining support. However, we believe options do not just come down to either having faith in a future success or endorsing skepticism. In this paper, we aim to redirect the discussion of the problem by shifting the focus of attention from strict definitions (in terms of necessary and sufficient conditions) towards a philosophical framework that allows conceiving of living systems as a natural kind, but whereby natural kinds are not to be defined by fixed necessary and sufficient conditions. We argue for a property-cluster kind approach according to which living systems constitute a natural kind with vague boundaries, capable of changing, and whose members do not need to instantiate every property. We draw from Boyd's homeostatic property-cluster theory and introduce two modifications, one regarding homeostatic mechanisms and another related to the scientific role of kinds. Thus, our view overcomes some difficulties of Boyd's theory and we are able to account for the natural kindhood of living things. We also emphasize the most appealing features of our approach for specific research fields and address three objections to this sort of approach.
\end{abstract}

Keywords Definition of life · Natural kinds · Boyd · Essentialism · Origins of life · Virus

María J. Ferreira Ruiz

mariaferreiraruiz@gmail.com

Jon Umerez

jon.umerez@ehu.eus

1 Department of Philosophy, University of Geneva, 5, Rue de-Candolle, 1211 Genève 4, Switzerland

2 Department of Philosophy, University of Buenos Aires - CONICET, Buenos Aires, Argentina

3 Department of Logic and Philosophy of Science, University of the Basque Country, Avenida

Tolosa, 70, 20018, SAN SEBASTIAN, Gipuzkoa, Basque Country, Spain 


\section{Introduction}

Despite the remarkable amount of biological knowledge gathered to date, questions such as "What is life?", "What is a living system?" and "What is the difference between living and non-living systems?" remain unsettled. There hardly is a better example of an old and intrinsically philosophical issue that is in constant renewal by current scientific developments. In some people's opinion, there is a sense of life of an intuitive nature: "... we have an effective enough intuitive or pragmatic notion of life" (Umerez 1995, 78). Jean Gayon has recently strengthened this assessment explaining in detail why life is "a primarily intuitive notion" based on a cognitive ability derived from evolution and perfected through learning and scientific practice (Gayon 2010, 232-233). Here, however, we are concerned with the scientific notion of life.

Traditionally, the notion of life has been displayed in biology textbooks or monographs in the form of a series of distinct but overlapping lists of different properties intended to capture its nature (Mayr 1982). These lists (see also, i.e., Farmer and Belin 1992, Emmeche 1994) feature properties or criteria that, when taken one by one, are problematic for a number of reasons; they are either threatened by counter-examples, the existence of "grey cases" (possessing some typical features of living systems but lacking some others), turn out to be unpractical, or are at odds with theories in origins of life research. This situation has compelled many authors to discard lists and try to provide proper definitions. Those definitional attempts become apparently more imperative when recent theoretical and technical advances within some specific fields, including artificial life, synthetic biology and astrobiology, pose the traditional question 'what is life?' in a new fashion and confronting more ambitious challenges. The space of possibilities that these research programs open for life beyond (as has been put) lifeas-it-is-now, that is, life-as-it-initially-was and life-as-it-could-be, is quite thrilling (Langton 1989, 1). It seems that some definition of living systems is assumed, but also requested and even challenged by these fields. In those areas, the adequacy of the lists of properties is subjected to harsh criticism and a request for a proper definition becomes more and more prominent. Such a hypothetical, desired definition is expected to answer to diagnostic or demarcating purposes, and to support a claim of universality transcending the allegedly particular case of present natural life on Earth.

It is now generally agreed that none of the traditional conditions for life are necessary or sufficient, leading some to fairly conclude that living systems cannot be defined. Yet, we may still observe that, in many cases, at least, "[B]iology, even if it lacks a precise and unanimously agreed upon definition of life, is able to use those lists of properties in a very coherent and almost unproblematic (unanimous) way when it is needed to identify a system as alive or not" (Umerez 1995, 78-9). We agree with the skeptics that living systems cannot be defined by a set of necessary and sufficient conditions. However, we believe it is not impossible to give a general account of living systems. Indeed, the various properties used in biology to characterize living systems may be rendered into a proper and philosophically sound concept. In this paper, we propose a property-cluster kind approach to living systems in order to overcome the obstacles that stand in the way of a classical definition. Living systems, we argue, are better understood as constituting a natural kind whose members have at least some properties in common. Our defense of a cluster kind approach involves a modification of Richard Boyd's theory in two ways: first, we reject the requirement that an adequate account must identify underlying homeostatic 
mechanisms, and secondly, we suggest that the utility of natural kinds must be understood more broadly than only in terms of inferential practices. These modifications contribute to amend the restrictive character of Boyd's theory and to come up with a more naturalistic concept of scientific natural kinds.

The paper is organized as follows. In section 2, we review the problem of defining living systems leading to skeptical stances. In section 3 we review the traditional essentialist conception of natural kinds as well as Boyd's homeostatic theory. Having stated the problem and the general philosophical approach, we develop the thesis that living systems can be well understood as a cluster kind, as well as our modified version of property cluster kinds, in section 4 . In section 5 , we reply to objections, and in section 6 , we offer some concluding remarks.

\section{The problem of defining living systems - The past, the present, and the possible}

Different definitions of living systems can be found in textbooks and scientific articles, but are they good definitions? Moreover, are they all equally good? Definitions, in their most traditional sense, are a set of individually necessary and jointly sufficient conditions. A proper definition of living systems should have the form " $\mathrm{x}$ is a living thing if and only if [ $\mathrm{x}$ satisfies $n$ conditions]". Also, we would expect any valuable definition to do at least two crucial things: to cover the most paradigmatic instances and to serve as an operational criterion with which to discriminate between entities that meet those conditions and entities that do not.

As mentioned above, several criteria have been proposed to characterize living systems (i.e., Schrödinger 1944; Mayr 1982, 1997; Farmer and Belin 1992; Emmeche 1994; Luisi 1998; Koshland 2002; Dupré and O’Malley 2009; Ruiz-Mirazo et al. 2010; Benner 2010, among many others). Some of these properties are tightly interconnected or even partially overlapping. Among the most popular and discussed ones (although the list could be longer), we can mention:

- Cellular organization: the cell is the unit of life, the minimal structure that ensures vital activity.

- Metabolism: living systems engage in complex biochemical reactions through which free energy and nutrients from the environment are used to produce compounds needed to maintain their organization.

- A far-from-equilibrium state: it is characteristic of living systems that they resist increment of entropy though their metabolic activity.

- Autonomy: the general capacity of self-organization and self-maintenance of organization via metabolic processes during a certain period, without the need for constant external intervention.

- Spatial boundedness: a living system is to some degree physically and chemically circumscribed, delineating the system's environment from an external one (a requisite for a successful metabolism).

- Reproduction: the capacity to bring about a similarly constituted system that is capable of the same kind of processes. 
- Responsiveness: living systems have the ability to react to external stimuli and to defend themselves against injuries.

- A carbon-based molecular constitution: carbon is a versatile element, capable of binding in stable and at the same time complex and different compounds.

- Evolvability: perhaps the most important feature of life as we know it is its capacity to undergo Darwinian evolution, in virtue of its ability to reproduce with heritable errors that may result in fitness differences.

- Growth: the increase of size or number of parts, mostly by a regulated process. ${ }^{1}$

"List-definitions" of this sort are typical in textbooks and differ substantially from one another, although they frequently partially overlap. Proposed definitions of life derived from them tend to select a few properties that are argued to be more important. Very often, the relative weight given to particular properties is influenced by the field in which its proponents work. But while nobody would deny the significance of such properties, the question is how universal or essential they are. How do we know that the list is complete and representative? As far as we know, the above criteria could merely be idiosyncratic of living systems on Earth, which could well be an unrepresentative example of living systems, and the definition would be of no use outside this domain (this is known as the $N=1$ problem in astrobiology or the limitation of life-as-weknow-it in artificial life). This and other concerns have been discussed for some time.

\subsection{Counter-examples, borderline cases, Operativity, and theoretical conflicts}

The main concerns often raised in the literature come in three sorts: there are more counter-examples and grey cases than we can allow for, the lack of operativity of the Darwinian criterion, and the fact that a definition of living systems is at odds with theories of the origins of life, among others. Let us briefly examine them.

Perhaps one would expect issues to arise only in extreme, borderline or exotic cases; yet, interestingly, one often-cited counter-example to a criterion, namely, reproduction, involves what we would regard as very paradigmatic instances of living things. If the capacity to reproduce is a necessary condition for being alive, then it follows that any two dogs capable of mating are alive, whilst a single or an infertile one is not. Infertile hybrids, such as mules, would fail to meet this criterion as well. Conversely, this criterion may be met by "obvious" (to some, anyway) cases of non-living things. Particularly, it has been claimed, for example, that crystals reproduce and grow (Kondepudi et al. 1990; Benner 2010). ${ }^{2}$

Dupré and O'Malley (2009) have questioned the criterion of boundedness, often referred to as characterizing life, from their treatment of biological individuality. According to these authors, "the boundaries of a plant and animal are precisely the sites where complex interactions occur between entities generally considered distinct, but these interactions are so closely coupled that we are strongly tempted to see them as

\footnotetext{
${ }^{1}$ Philosophers of biology often disagree on the proper understanding of many of these concepts. This is especially true of autonomy, evolvability, boundedness, and reproduction, which star in their own debates. Here, we lack the space to engage in such debates, and will remain as neutral as possible. Our proposal in this paper, if valid, is so across the different interpretations of these properties.

${ }^{2}$ For other properties arguably shared by living systems and crystals, see Schulman and Winfree 2007 and Palacci et al. 2013.
} 
parts of the same system" (Dupré and O'Malley 2009, 12). In short, a focus on interactions makes it difficult to draw sharp distinctions between individuals and this, in turn, makes it difficult to talk about boundedness (a feature of individuals).

Metabolism faces a similar challenge, because it is typically a collaborative activity. A human is not capable of certain autonomous metabolic processes that are essential for survival: collaboration with bacteria is indispensable. But perhaps we should not demand that living things be capable of carrying out every essential (for survival) process. Maybe the mere capacity of some independent metabolism will suffice, with the obvious difficulties in pinning down what is meant by 'some' and 'independent'.

Viruses are an interesting case for evaluating the criteria. These nucleic acid-based compounds capable of replication, of mutation and, ultimately, of Darwinian evolution are generally regarded as mere "infectious sub-cellular agents" or "mobile genetic elements" (Alberts et al. 2002), in other words, entities beneath the level of the living. For mainstream biology, viruses are not living things because of their incapability of autonomous reproduction and metabolism, and their reliance on hosts' machinery (Lwoff 1957; Moreira and López-García 2009; Van Regenmortel 2007). ${ }^{3}$ However, Dupré and O'Malley's remarks on interactions show that the general lack of independence or autonomy of viruses is not really exclusive to these entities. On the other hand, the Alcidianus two-tailed virus exhibits development outside the host cell as it can grow tails independently of its host's resources (Häring et al. 2005). In turn, mimiviruses (the largest known viruses) are now puzzling virologists due to their size and the characteristics of their replication (Claverie et al. 2006). Furthermore, during cell infection, when the only genome expressed is the viral one, it could be argued that a cyanobacteria ceases to be a bacterial cell and becomes a virus with cellular appearance (Forterre 2010). Some viruses infect other viruses (virophages); if, like cells, a virus can itself be infected, then perhaps the difference between them is not so clear-cut (Pearson 2008).

Additionally, organisms or parts of organisms may be considered to be neither alive nor dead in certain circumstances. For example, most of the properties of living systems are not observed in frozen organisms and dried seeds while in such states (Gánti 2003).

On the other hand, a very important criterion of living systems (the one in the light of which everything in biology makes sense!) fails to be, as many have pointed out, operational in the search for new forms of life. Evolution requires long-term and statistical (population-level) observations. As Luisi remarks, Darwinism leaves out life-here-and-now (synchronically observed) and is at odds with the reasonable demand that a definition must be operational. These are in fact the problems faced by NASA's working-definition of living systems, according to which life is a self-sustained chemical system capable of undergoing Darwinian evolution. As Luisi has pointed out "it would be difficult to apply it to an individual blob found by space explorers in another planet. Even if they were to find a colony of the things, should they wait perhaps a few million years to observe the Darwinian evolution?" (Luisi 1998, 618). A way out of this conflict is to make a modal amendment to the criterion and claim that we do not need to make actual long-term observations, but merely be able to recognize chemical systems capable (in principle) of undergoing Darwinian processes. Steven Benner has explored what the requirements for a chemical compound (other than our familiar DNA/ RNA) would be in order to support Darwinian evolution (Benner 2010). However, in the

\footnotetext{
$\overline{3}$ There are other kinds of reasons as well (see Moreira and López-García 2009 and Brussow 2009).
} 
event that such a compound be found, its mere observation would not be decisive, for its "Darwinian potential" would still be presumptive. Worse, the criterion would not settle things on its own, for we still might ask whether a virus is alive, and the answer will depend on the weight given to the evolutionary criterion (Luisi 1998).

A final concern arises from the theories about the origins of life. The most favoured picture of the origins of life is a continuum from low to higher complexity molecules (Luisi 1998). And for pioneer biologist Joshua Lederberg, a strict definition of living systems would posit an arbitrary qualitative crack at some point (Lederberg 1960). It is still a matter of debate as to what "stages" came first in this gradual process (enzymatic activity or self-replication?); either way, a strict definition of living systems and a gradualist theory of its origins are incompatible.

The Darwinian criterion is also problematic for origins of life research. Robert Pennock points out to the problem that taking evolution to defining life might not be very insightful in origins of life research, since Darwin's theory aimed at explaining the origin of species, not the origin of life. Therefore, "evolution would not be relevant to explaining the origin of life except insofar as the two mean the same thing". (Pennock 2012, 16). However, some have argued that living systems may have passed through a period during which there was reproduction but not Darwinian evolution yet. This is the "protein-first" or, more generally, "metabolism-first" hypothesis: protein-based entities capable of metabolism but not capable of replication preceded nucleic acidbased replication (Dyson 1985; Cleland and Chyba 2002). Of course, not every theory of the origin is equally favoured, and some would consider metabolism-first theories to be wrong precisely because said entities could not undergo Darwinian evolution. But, as has been astutely pointed out, such a conclusion presupposes that living things must be capable of this (Diéguez 2013). Given that the puzzle of the origins remains unsettled, we do not want a definition to constrain our hypotheses on the matter. If anything, it would make more sense to have our theories of the origins influence our definition of living systems.

\subsection{Skeptical conclusions}

As we have seen, several problems stand in the way of finding a precise and strict definition of living systems. Thus, some authors have concluded that living systems cannot be defined at all, or that the very quest for a definition is pointless.

Carol Cleland and Christopher Chyba (2002) have argued that a proper definition of living systems depends on there being a theory of the nature of living things, i.e., a "theory of life." They compare the situation of living systems with that of water before the molecular theory. Only once we achieved an understanding of the molecular structure of matter, were we able to define water as $\mathrm{H}_{2} \mathrm{O}$. Analogically, a theory of life would allow us to define living systems in an (fairly enough) unambiguous way. However, we lack such a theory, and perhaps it is ultimately unattainable. In Cleland's opinion, then, the definitional approach to living systems is "fundamentally misguided" (Cleland 2012).

Others believe that the possibility (or not) of finding a definition is not actually the main issue. Edouard Machery (2012) argues that attempting to define 'life', in a scientific sense of the term, is (even if possible) rather pointless. He supports this idea by showing that different biological disciplines favour different notions of living systems. Guided by their particular interests and goals and by the phenomena each 
investigates, astrobiologists, molecular biologists and "origins"-biologists may put differential weight on certain properties. Moreover, there is no consensus even within a given discipline. So, Machery concludes, it is unlikely that all disciplines will converge on the same definition.

Naturally, the alleged issues considered above can be discussed further. Here, we can only point out that regardless of whether or not they succeed in showing that scepticism is well motivated and that the pursuit for a strict definition has to stop, they are at least sufficient to motivate alternative approaches. In this sense, it is worth stressing that, in parallel to the problems we have just discussed, it is true that lists of properties of this sort have been used effectively in the biological sciences, which suggests that they cannot be simply dismissed as flawed. In this context, we believe that there is another way to gain a better view of the complex and diverse phenomena roughly encompassed by the term 'life'. Taking this path implies moving away from strict definitions and advocating for a rather different approach to the problem, namely, a philosophical, natural kind account of the notion of living systems. Before developing our account, we will briefly review the general background on natural kinds in philosophy.

\section{Classic natural kinds and property cluster kinds}

The topic of natural kinds is a classic one in metaphysics and philosophy of science. We commonly believe that objects in nature can be classified into different kinds of things, and that natural scientific theories and disciplines talk about these kinds. Stances on the naturalness of kinds (are there genuine divisions in nature?) range from realism to conventionalism. A realist believes that there are genuine divisions existing independently of human practices, even if we reflect them incorrectly. In contrast, a conventionalist stands for the idea that all kinds are mere artifacts reflecting our needs and interests, with no existence of their own.

According to the traditional conception, natural kinds are defined by a set of individually necessary and altogether sufficient conditions (a conjunction), which constitute the kind's essence (Putnam 1973; Kripke 1980). Essential properties are intrinsic features, typically, to be found in the thing's micro-structure. Such properties are possessed by all and only the members of the kind, and they must explain why members share other superficial features. For the most interesting scientific cases, such a definition was hard to achieve, and this traditional conception received extensive criticism (see Bird and Tobin 2015 for a summary). Chemical species and substances and biological species were essentialistic philosophers' preferred examples. Yet, philosophers of chemistry have argued that chemical species and substances are not correctly captured by the essentialist view of natural kinds (Needham 2000, 2011, van Brakel 2005; Ruthenberg 2012). In philosophy of biology, many authors concerned with biological species also turned against the traditional view. It was argued that an essentialist conception of species is precluded by the "population thinking" that characterizes modern Darwinian biology, so any essentialist view of natural kinds is unsuited for biological kinds (Mayr 1966, 1976; see also Sober 1980). Objections against the essentialist view of natural kinds were not only motivated by a general rejection of natural kind talk in biology altogether (such as the view that species are spatio-temporally extended individuals rather than species, cf. Ghiselin 1974 and Hull 1978). Rather, essentialism was contested even by advocates of the natural kindhood of biological species. 
In several philosophers' view, a different approach to natural kinds was better prepared to account for species. Richard Boyd (1991, 1999) and others (for example Millikan 1999; Griffiths 1999; Wilson et al. 2007) have extended and modified the traditional view of natural kinds and advocated for the notion of homeostatic property cluster kinds (HPC), that is, kinds not definable in terms of sets of necessary and sufficient conditions but in terms of a cluster of (a generally large number of) co-occurring properties. Some have considered that the impossibility of defining a kind thusly is a sign of convention: that which cannot be defined by necessary and sufficient conditions is not a natural kind (Hacking 1991), but Boyd has argued against this idea. According to him, HPC-kinds share some of the features of the traditional (essentialistic) kinds. For instance, it could be shown that they are not social conventions (they are defined a posteriori) and they serve in inferences and explanations. Simply put, a HPC-kind is any potentially loose family of co-occurring properties that may be employed in scientific inductions and explanations.

Members of HPC-kinds are not required to instantiate every property in the cluster, so determining membership is not a simple task. The clustering is somewhat contingent, but only to the extent that it is not obtained from an essence, and not in the sense that it cannot be accounted for. Boyd suggested that the properties are held together in a stable range either because the presence of one favors the presence of another or because there are underlying homeostatic mechanisms that tend to maintain their presence. Thus, HPC-kinds are defined and individuated by means of some or all the properties in the cluster in combination with some or all the mechanisms responsible for the co-occurring clustering of properties.

HPC-kinds have an interesting feature. As environmental contexts may change, so may the cluster. Thus, no property is individually necessary for membership. For the same reasons, the set of underlying mechanisms is subject to change as well. This tolerance to change allows the boundaries of the kind to be vague. Such flexibility is responsive to the fact that things are not neatly divided in nature. But, importantly, this flexibility does not compromise heuristic or epistemic potentiality. According to Boyd and followers, it is the existence of underlying mechanisms that is responsible for the kind's epistemic potentiality for inferential practices, that is, what endows the kind with projectibility (Kornblith 1993; Millikan 2000; Slater 2015).

In short, a group of things constitutes a HPC-kind when:

i) there is a family of co-occurring properties,

ii) held together by certain mechanisms, and

iii) used in inferences and explanation.

Importantly, and unlike essentialistic natural kinds, HPC-kinds may also (a) be capable of changing, and (b) have fuzzy boundaries. While these two extra features are not conditions for natural kindhood, they may be relevant for the most interesting cases (such as biological species). In fact, HPC's tolerance to change and fuzziness is largely responsible for its appeal amongst philosophers of science.

In particular, the remarkable tolerance of HPC theory to change, fuzziness and exceptions seems to make it suitable to account for living things as a natural kind. However, certain features of HPC theory are restrictive or problematic, and need to be discussed. In the next section, we discuss the problems with HPC theory, put forward our modified property cluster kind view, and apply it to living systems. 


\section{Living systems as a property-cluster kind}

From our perspective, the impossibility of achieving a strict definition of living systems and the empirical nature of the problem suggest that living systems must be conceptualized in a framework capable of admitting blurred boundaries and retrieving the continuity and smoothness to be found in the transition from inorganic to organic systems. It would also be desirable for the account to do justice to the actual use of lists of properties in biological practice we mentioned in Section 2. A further desideratum is that this should be achieved without compromising a naturalistic stance (that is, we want to remain close to relevant scientific practice) and without dropping a very modest realist stance (that is, that allows for the possibility that we may make mistakes). In this section, we will show how our non-essentialistic natural kinds-framework fits these requirements.

Evelyn Fox Keller has argued, from a Foucaultian point of view, that life is not a natural kind because the concern with the definition of life is historical and the question is to be answered accordingly $(2002,294)$. We think she misses the point. On the one hand, every human question is historical; on the other, we wonder to what extent life is different from any other putative example of a natural kind (however understood) in this sense. Surprisingly enough, the idea that life is a natural kind has barely been defended explicitly. In attempting to characterize the notion of biological organism, Robert Wilson (2005) took a HPC approach. In his view, organisms are a HPC sub-kind of the kind of living things. Yet his account says very little about one key feature of the HPC view, namely, the underlying homeostatic mechanisms. Wilson's account is interesting in that it applies HPC to biology very broadly, and it conveys tools to deal with other disputes in philosophy of biology. However, since homeostatic mechanisms play such an important role in HPC theory, it is reasonable to require that the set of relevant homeostatic mechanisms be identified when offering a HPC account of living things, which in Wilson's account are simply assumed to exist (but again, his main concern was the notion of organism more than that of living things). Antonio Diéguez (2013) has also argued that living things constitute a HPC in Boyd's sense. His argument is a reductio ad absurdum: if life is not a natural cluster kind, then it must either be (a) a folk concept, (b) a conventional kind, or (c) an individual. As none of these is the case,${ }^{4}$ then we may conclude that living systems are a HPC. Diéguez understood that more fruitful and interesting discussions are raised beyond the definitional approach, thus we share the overall spirit of his account. These are valuable

\footnotetext{
${ }^{4}$ Diéguez's argument to rule out the possibility of life being an individual is not entirely convincing, though. It runs as follows: elements of an individual enter part-whole relationships, whereas elements of a kind enter membership relationships. Thus, assuming that terrestrial life is an individual, if we encounter extraterrestrial life, would we regard both as parts of the same whole (namely, 'life') or as parts of two distinct individuals (namely, 'terrestrial life' and 'extraterrestrial life')? He points out that our intuition depends on previously determining whether the two samples have a common origin or not. According to him, we would feel more inclined to regard them as parts of the same individual in the case we stand for common origin, and this will be less likely if we stand for independent origins. Diéguez's favors the latter option, concluding that extraterrestrial and terrestrial life do not enter the sort of mereological relations to be expected from individuals. Therefore, 'life' cannot name an individual. However, this is not correct. The fact that terrestrial and extraterrestrial life are (ex hypothesi) not part of the same individual does not mean that they are not two different individuals. At best, it means they are not the same individual. We thank an anonymous reviewer for pointing this out.
} 
antecedents, ${ }^{5}$ but we believe a cluster kind notion of living systems must be explored further and somewhat differently -hopefully, both Wilson and Diéguez would agree with our arguments here. More specifically, some of the main requirements of HPC theory have been recently discussed and criticized, and such discussion has not, perhaps for simple chronological reasons, echoed their accounts. If we are to take a cluster kind view of living systems, we first need to address these recent concerns.

\subsection{Concerns about HPC theory}

Certain features of HPC theory have been argued to be either too restrictive or problematic, which severely affects its range of application. This is the case of two of the main the conditions of HPC theory, namely, underlying homeostatic mechanisms (ii) and inferential potentiality (iii).

Marc Ereshefsky and Thomas Reydon (2015), Reydon 2009) analyze several sorts of kinds that are not picked out by HPC theory, such as "functional kinds" (for instance, gene kinds) and "non-causal kinds" (exemplified by the phylo-phenetic species concept in microbiology). They argue from these cases that the presence of homeostatic mechanisms is not a sine qua non condition of kindhood. Microbiologists classify their organisms following certain that do not involve causal considerations. Functional kinds, on the other hand, are identified primarily by, precisely, functions, and functions, we know, are multiple realizable. In these cases, they say, scientists aim to find stable and reliable kinds with no intention of capturing their causal structure. ${ }^{6}$ One could think that the relevance given to underlying mechanisms in HPC theory may have been exaggerated, but the truth is that Boyd and followers never meant to claim that HPC theory picks out every kind there is and, therefore, cases that HPC theory cannot account for are not fatal to the theory.

$\mathrm{Be}$ as it may, there are still other, more theoretical concerns about HPC theory. For one thing, one could be wary of the very role of homeostatic mechanisms. For some, they are old essences in a new dress (this is how Häggqvist 2005 seems to read HPC Theory). But the most detailed and profound revision of HPC in this respect is due to Matthew Slater $(2013,2015)$. He has recently argued, against Boyd and his followers, that appealing to homeostatic mechanisms in a cluster theory of kinds is neither sufficient nor necessary to ground our epistemic practices with natural kinds, even in so paradigmatic a case as that of species as kinds. This is not a trivial matter, given that mechanisms in Boyd's theory are there to save the epistemic performance of natural kinds once essences are abandoned. On the one hand, Slater points out the difficulties to generate objective distinctions among

\footnotetext{
${ }^{5}$ Other somewhat related antecedent is Pennock's (2012) Wittgenstenian approach.

${ }^{6}$ We do not take the authors to claim that, in these cases, the underlying structure is irrelevant in absolute terms (nor do we believe this ourselves). First, they claim that HPC fails to pick out certain cases, which is not equivalent to claiming that it picks out no cases at all. If there is a case correctly picked out by HPC, then the causal structure is relevant for it. Secondly, claiming that knowledge and reference to the underlying causal structure is irrelevant for objects classified on different grounds is not equivalent to claiming that it would be irrelevant in other respects (that is, beyond the purpose of their classification). Certain differences among members of, say, a functional kind may be explored by looking at underlying causal factors, without this being in conflict with the fact that they had been previously classified by following criteria other than underlying causal mechanisms.
} 
kinds on the grounds of individuating mechanisms, since champions of mechanistic explanation such as Carl Craver acknowledge the inevitable human, conventionalist input needed for individuation. Secondly, he argues that in many cases (such as cell types) one would not plausibly regard the responsible mechanisms as the same-self; rather, "the relevant mechanisms would presumably be distinct tokens of the same type" $(2015,387)$. In this case, one might fairly want to classify the mechanisms themselves into different kinds: kinds of mechanisms. The co-instantiation of mechanisms might be explained in HPC terms by appealing to yet another set of mechanisms, thus over-multiplying our kinds and risking regress. This too is already noted by Craver. Finally, Slater argues that the mere existence of a homeostatic mechanism responsible for clustering some properties does not by itself ensure stability, since not every such mechanism operates constantly, or in every condition; oftentimes, they work under certain circumstances and stop working under others. Thus, further elements must be cited as explaining or ensuring the stability of a cluster. In short, HPC theory tends to overestimate underlying homogeneity.

Therefore, Slater proposes to change the focus from essences/mechanisms to their effect, namely, stability. He proposes the notion of "stable" instead of "homeostatic" property cluster kind. The question then becomes one concerning stability, by virtue of which kinds are apt for inferential practices, regardless of what causes such stability. In his view, patterns of co-instantiation are to a certain extent stable, that is, over a set of counterfactual conditions (Lange 2000) and this is what grounds their inferential potentiality-nothing else is required. The idea is that a property cluster $\Phi$ is stable when it is robustly the case that, for many sub-clusters $\varphi 1, \varphi 2, \ldots$, if a particular $\mathrm{x}$ has $\varphi 1$, then it probably has $\Phi$ (and so on with other sub-clusters). Robustness here amounts to such conditional's invariance under a range of counterfactual suppositions. Following Lange's treatment of scientific laws, Slater requires such counterfactuals to be consistent with the probabilistic entailments relationship from sub-clusters to clusters (i.e., we should not expect a fact to remain invariant under the supposition of its negation), compatible with natural laws, and to meet the relevant applicability standards (for example, be manipulable in contexts in which this is relevant). Importantly, "how much stability" does not seem to be the relevant concern in this view, which is largely relegated to the epistemic context. In short, he argues that mechanisms are not necessary; we just need to address stability. Cluster stability may well respond to homeostatic mechanisms, but this does not need to be the case. This view could be called "bare stability" by analogy with Häggqvist's "bare projectibilism" (more on this below). In general, we think that caution is sufficiently grounded as to favor a stable over a homeostatic viewpoint.

The requirement that kinds be projectable (iii) is not that simple either. While some have addressed Boyd's requirement, arguing that natural kind concepts indeed support inferences, others are less indulgent to such a philosophical assumption. As to the first case, Reydon argues that even functional kinds, which are often contrasted to more "standard" kinds (such as HPC kinds) because of an alleged difference in their explanatory value: reference to HPC kinds is considered to be explanatorily more powerful than reference to functional kinds. Yet, he argues that gene kinds such as 'hedgehog' are functional kinds that still play a clear role in mechanistic explanations within molecular biology: "Reference to the 
functional kind to which a particular part of a system belongs is explanatory as the basis of a generalization about the behavior that it is expected to exhibit when placed in a particular environment" (Reydon 2009: 731).

Others, however, reject the presupposition entailed by HPC theory that projectibility can only be ensured by homeostatic mechanisms and the presupposition that these are decisive for the kind's reality. In Sören Häggqvist's view, "what matters is the reality of the kind, or, to put it another way, its genuine, as opposed to apparent, projectibility" (2005, p. 80, emphasis in the original). The "bare projectibilism" view entails that for the existence of a kind it does not matter why it is projectible or how we know it is projectible. What matters is projectibility itself. Thus, he argues that "we should accept the legitimacy of kinds exhibiting, as it were, brute projectibility," meaning, "kinds that are projectible even though we don't know of any underlying mechanism that explains why they are projectible. It should be kept in mind that "brute", in this context, is a term of epistemology: there may in fact exist a mechanism that would, and perhaps in time will, explain the projectibility of the kind. The suggestion is that we cease to stake the kind's status as a genuine kind on the outcome of our search for such a mechanism" $(2005,81)$. His main argument in support of this idea is that we have many examples of projectible categories, such as that of electron, whose projectibility are not accounted for mechanistically. Moreover, he claims that many categories are projectible independently of our confidence that such a mechanistic explanation will be achieved in the future.

Yet a different observation on projectibilism is its holistic character. In discussing species as natural kinds, Miles MacLeod warns that:

Knowing what features of an organism are generalizable in given circumstances takes a lot of knowledge that cannot be reduced to, say, a particular generic set of mechanisms that explains why properties generally cluster. Researchers need theory, experimental testing, and statistical sample analysis to tell them that the particular features they are dealing with are projectable in the particular environmental and other contextual circumstances given knowledge of their biological variability, function, and the like. Such mechanisms as natural kind theorists envisage, such as gene exchange, may be necessary but not sufficient conditions in this respect for determining which properties might be projectable" (2013, 111-112).

This is an observation that takes considerable pressure off natural kinds or natural kind candidates, since it entails that projectibility is not a feature of natural kind concepts alone, but something rather distributed along epistemic objects and contextual circumstances.

Even though Slater is less concerned with casting doubts on the requirement of projectibility than he is on rejecting the requirement of underlying mechanisms, he warns that

A theory of natural kinds was never (or should never have been) meant to provide blanket inductive license to project properties associated with a kind to an individual possessing some of those properties. Rather, it helps us understand 
how certain categories do serve this role when they do. It is well known that inductive inference occurs only against the backdrop of background knowledge. This background can defeat the prima facie epistemic warrant provided by a category's kindhood. (2013: 178)

One can even go further and reject the idea that natural kinds must be projectable at some point, altogether. So instead of taking for granted the idea that natural kinds merely (and primarily) serve inferential purposes, others insist on showing how diverse scientific practices with natural kinds are. Ereshefsky and Reydon's examples mentioned above are not only examples of kinds whose causal structure is irrelevant (for a given research field where the classification system is produced) but in fact they exemplify different ways of using kinds successfully. They claim that a truly naturalistic account of natural kinds should reject the "limiting assumption that all scientific classifications ought to support inductive practices" (Ereshefsky and Reydon 2015, p. 985).

The points just explained are general concerns about HPC theory, but we want to point out in which ways they are relevant for our case study. Consider, first, the reasons that would seem to motivate a HPC approach to life in the first place. Well, one would have to admit that living systems are indeed characterized by a large family of properties that tend to co-occur, which are subject to change, as entailed by evolutionary theory and theories of the origins of life. And one would also have to admit that the concept of life seems to be fuzzy indeed, as evidenced by long-standing controversies amongst biologists over prions, viruses, and so on. HPC's tolerance for change and fuzziness is consistent with the smooth, non-discrete transition to life. In sum, it seems that the very reasons why defining life is so difficult can be taken as pointing out to a HPC approach to the matter.

Yet, things may start to fall apart when considering requirements (ii) and (iii), that is, that the family of co-occurring properties be held together by certain homeostatic mechanisms, and that the concept of the group of things having such properties be used with inferential purposes. For one thing, and fundamentally, concerns could arise from the fact that we are dealing with a highly heterogeneous group of entities and properties here. Such heterogeneity makes it very unlikely that there be one single set of shared homeostatic mechanisms responsible for the co-occurrence of those properties. Moreover, many aspects of living systems in fact are believed to be closer to "frozen accidents" than the effects of a persisting homeostatic mechanistic cause. It could be argued that such heterogeneity and contingency pose no problem for HPC theory, as it allows the relevant mechanisms to be multiply realizable (this possibility is considered in Wilson et al. 2007 in addressing species, genes and stem cells). However, the very way in which philosophers conceive of multiple realizability implies a certain notion of identity. At the risk of stating a tautology, a multiply realizable mechanism is $a$ (multiply realizable) mechanism. Our concern is valid for the general or type mechanisms as much as it is for their implementations or tokens. On the other hand, as a consequence of HPC's requirements, we are incapable of distinguishing between two very different situations: the one in which something is just not a cluster kind and the one in which we simply fail to acknowledge its natural kindhood because we do not know anything about the underlying homeostatic mechanisms (perhaps, not yet). This difference could be of a special importance for research fields in which there is still 
much that we don't know, such as astrobiology. Lastly, apart from considering that homeostatic mechanisms are just the old essences in a new dress, a commitment to the existence of (unknown) homeostatic mechanisms clustering the properties of living systems can be excessive. We may not know what tends to keep this clustering and whether it is a homeostatic mechanism (or set of mechanisms). This is not a declaration of causal skepticism; admittedly, certain causal factors are responsible for the combination of properties we observe in a given system. All we are claiming is that, first, causation does not necessarily take a homeostatic-mechanistic form, and second, if we can't identify homeostatic mechanisms responsible for the clustering of all living things, then, how can we grant projectibility (iii)?

In our view, the issues discussed so far do not entail that we should throw the baby out with the bathwater. HPC as it stands may not be entirely satisfactory, but the abovementioned concerns can be translated into a revised and improved version of an account of cluster kinds. Slater's stability account, while overcoming concerns about HPC, still place a high value on projectibility as the only relevant epistemic role for natural kinds. Drawing inspiration from the above-discussion, we now make the case for an alternative concept of cluster kinds and we show living systems to meet its criteria.

\subsection{A modified version of property-cluster kinds}

As follows from discussions in the previous section, requirements (ii) and (iii) in HPC are, in general, either restrictive or problematic. It seems that living systems fail to satisfy HPC's criteria mainly because there is no obvious way to specify the underlying mechanisms clustering the shared properties of living things. Bare projectibilism and bare stability do a fair job in arguing against requirement (ii), but they remain too committed to requirement (iii). It seems that life will also fail to accommodate to these views because there is no obvious way to show the inductive potential of the concept either. Does this mean that we are forced to discard a cluster kind approach? Or, moreover, does it mean that we need to give up any attempt to conceiving of life as a natural kind at all? We don't think so. As discussed in the previous section, we do have enough reasons to revise HPC theory, but we will argue that this does not compel us to deny the natural kindhood of life. Thus, we propose the following modified version of propertycluster kind $\left(\mathrm{PCK}^{*}\right)$. A group of things constitutes a $\mathrm{PCK}^{*}$-kind when:

(i) there is a family of co-occurring properties,

(ii) that display a relevant degree of stability (over a range of counterfactual conditions)

(iii) and that play some role in scientific practice (epistemically and heuristically).

PCK*-kinds may also (just like in "standard" HPC), be capable of changing, and have fuzzy boundaries.

PCK* does not inherit the difficulties regarding homeostatic mechanisms from HPC and is less limited than HPC regarding the usefulness of kinds in scientific practice, both discussed in the previous section. Now, let's examine whether or not life meets PCK*'s conditions. 
As to stability, we followed Slater's idea of patterns of co-instantiation that are to a certain extent stable, that is, invariant over a set of counterfactual conditions. First, recall that that co-instantiation, as we have already said, is not, and needs not be always perfect. It will be the case that particulars of certain natural kinds exhibit different patterns of instantiation. In the case of living beings, this would be both due to the fact that living systems can be very heterogeneous and to the fact that they can assume a variety of states over their lifespan. For example, metabolism and spatial boundedness are not always co-instantiated, as in the case of viruses (this might be the case with Alcidianus, but arguably is not the case for others); or seeds, for example, would exhibit a certain pattern of co-instantiation in their dormant state and a different one under other circumstances. This illustrates the point that the cohesiveness of a given combination of properties varies from system to system. Under a cluster view of kinds, just as members need not to instantiate every property, patterns of co-instantiation cannot be expected to be invariable. We found it unproblematic to admit that different research areas may put differential weight both on specific properties and on their cohesiveness, because it may be that certain counterfactual conditions be of no interest in a given research context (for example, it might be that a field be only concerned with variables they can manipulate (Slater 2015, 401). So, different degrees of instability may be tolerated in different disciplines. But then, which is the relevant range of counterfactuals under which a cluster needs to be stable to be able to count a kind as a natural kind? This is not a question that admits of a definitive answer, either in Slater's view or ours. In particular, it does not admit of an answer that implies drawing a clear-cut line between, say, "too unstable" for a natural kind, and "stable enough" for a natural kind, just on the grounds of the degree of stability alone. In our view, the condition of stability must not be disassociated from $\mathrm{PCK}^{*}$ other condition that the putative kind plays epistemic and heuristic roles in scientific practice.

On the other hand, our understanding of the successful use of kinds is broader than in HPC Theory, but also broader than in Häggqvist's bare projectibilism and Slater's bare stability, who reject the need for homeostatic mechanisms to ground projectibilism and to ground stability, respectively, without much questioning of the received view that natural kinds are, above all, projectible categories. Kinds may be deployed, in a useful manner, both epistemically and heuristically. Synthetic Biology, Artificial Life and astrobiology are good grounds to appreciate the heuristic potentiality of a cluster notion of living things. The many properties in the cluster are useful as guiding notions abstracted or generalized from known and familiar forms of life (Umerez 1995). Indeed, this utility is revealed and tested, on the one hand, in the attempts to create life from scratch using biochemical building blocks, or based on a different material (non-carbon) or even without a material realization (computationally-based); as well as, on the other, in the search for extraterrestrial forms of life elicited by very minimal, dissimilar and controversial evidence, serving as clues to detect, identify or designate instances of living systems beyond the safe domain of life on Earth. These are activities that could hardly be reduced to inferential practices. And again, which sub-clusters (different combinations) of properties and which is the relevant degree of stability that would guide research may perfectly vary from one discipline to another, which is consistent with the basic idea of cluster kinds where boundaries are fuzzy and that priority cannot be assigned once and for all to any property because no property needs to be instantiated universally (more on this in section 5.3). 
Acknowledging non-inferential epistemic uses of the concept of life doesn't mean that inferential practices are impossible or unimportant. The concept of life can certainly take part in inferences and explanations as has been classically required from scientific natural kinds. Marc Lange (1996) exemplifies this by analyzing the historical controversy over the status of fungi. Whether fungi are living creatures was a puzzling matter until mid-nineteenth century. The debate was concerned with whether or not they display the typical features of life and, if so, whether the explanation for this was that they are living. Lange notes how fungi's vitality was in fact used to explain a very specific feature, that is, their internal organization. Diéguez (2013), in turn, suggests a hypothetical example for future astrobiology research: if we discover a planet with a considerable amount of atmospheric oxygen, we can infer that it is likely that the planet has life (or has had it in the past). However, it is not the case that science is all just about prediction and explanations; description and classification are a major task in science as well. Moreover, there are areas in biology in which prediction has a minor role (Dupré 1993; Mayr 1997), whereas classificatory, kinding practices are pervasive. An obvious and yet crucial epistemic role for kinds is to provide a useful way to classify and describe things. Relatedly, natural kind concepts are helpful for delimitating objects of study and application domains of disciplines or research fields, circumscribing a portion of phenomena of specific interest. This is particularly true for our topic here: the concept of living system actually performs such a domaindelimitation role for biological sciences.

In sum, the two major requirements of HPC, namely, projectibilism and the homeostatic mechanisms-grounding, are either restrictive or problematic. A few authors have challenged HPC and addressed concerns over projectibilism and concerns over the grounds of stability, but the two have been addressed quite independently from one another. PCK* is a better conception of natural property-cluster kinds as it overcomes the issues stemming from both projectibilism and homeostatic mechanisms. We do not expect our $\mathrm{PCK}^{*}$ view, however, to cover every possible case of scientific natural kinds. Just to mention an example, functional kinds have different features and are also very relevant in scientific practice (see Reydon 2009 for a nice defense of the epistemic role of functional kinds). What we have tried to pursue here is a better understanding of cluster kinds in the light of various criticisms to HPC theory and inspired by the debate over life.

\subsection{Virtues of this approach}

Thus, we have argued that life meet $\mathrm{PCK}^{*}$ 's conditions. Now, we want to show why a $\mathrm{PCK}^{*}$ approach to life is particularly interesting in regard to the issues mentioned in Section 2, namely, controversial known cases, new evidence, research in astrobiology, artificial life and synthetic biology, and the origins of life, where the critical differences between a definitional or essentialistic approach and ours become evident.

First, it succeeds in reconciling, within a single scheme, controversial known cases (such as viruses or prions) with non-controversial ones. Grey or doubtful cases of life-here-and-know, rather than being counter-examples (as for a definition), are to be expected and entail no conceptual problems. There is no need for a different category of things, and thus we gain a more cohesive picture of biological phenomena. Moreover, a conception of living systems as a $\mathrm{PCK}^{*}$ kind is the 
only one capable of accounting for a feature of scientific practice normally neglected when addressing the problem of the concept of living systems, namely, biology's very object of study. It is assumed that a great variety of things are a matter of biology, regardless of their explicit status as living or non-living things. Viruses, for instance, are generally regarded as non-living things, yet they are studied within biological frameworks: a considerable amount of biological effort is invested in studying their evolution, taxonomy, genetics, ecological interactions, etc. (Forterre et al. 2014; Manrubia 2012). Similarly, techniques and analysis from proteomics are applied to understand prions just as organismal, i.e., living things' proteins are studied (see for example Campisi et al. 2015). Darwinian tools are also applied to understand their capacity to adapt and evolve (Li et al. 2010). Of course, the fact that a certain entity is approached with biological epistemic tools does not imply that said entity is, for that very reason, a biological, living thing. Oftentimes, epistemic tools borrowed from a certain discipline are applied far beyond that discipline's intended domain, without this having any ontological consequences for the object by itself. However, there is an intuitive difference between studying viruses and, say, financial markets from a biological point of view. Even if someone goes on to claim that financial markets are complex adaptive systems that may be alive (see Bedau 2007, 458-459) rather than considering this a mere epistemic convenience, still we doubt anyone would regard virology as endowing biological epistemic tools with a mere instrumental use when applied to viruses. Thus, without rendering everything that is amenable to a biological approach a living thing, our proposal bears the rationale for the fact that this intuitive difference between financial markets and viruses appears to make sense. This can only be the case when one comes to terms with continuity in nature (more on this below). Asking whether or not, at the end of the day, is $X$ alive means not accepting the very terms of our continuistic approach. Biology, in short, studies a natural kind, where rarities and grey cases find their place in a non-disruptive scheme.

Secondly, as this is not a strict and a priori definition, the cluster is revisable in the light of new empirical evidence. As investigation progresses, new properties of living systems could be discovered and added to our knowledge of the cluster, and new patterns of co-instantiation can be discovered, without major conceptual difficulties (unlike the difficulties this would embody for a definition in terms of necessary and sufficient conditions, as well as for an essentialistic natural kind view).

A third important concern is how we can understand living systems so that this notion does not blind or constrain research in fields such as astrobiology, artificial life or synthetic biology. In our view, a $\mathrm{PCK}^{*}$ conception of living systems induces no blindness because it does not compel us to look for, or to recreate in silico or engineer in vitro objects exhibiting a fixed set of properties, as a strict definition would do. The properties in the cluster have a strong heuristic rather than a constraining power in our account. Thus, for example, artificial life researchers would value a cluster conception that motivates research while dropping the expectation of achieving an artificial instantiation of the essence of life. In the case of synthetic biology, it is important that a definition of life does not constraint the space of possibilities in the design and synthesis of novel genetic circuits, entire genomes, or even entire organisms (Malaterre 2009). Similarly, in astrobiology, the conceptualization of life must work in a way that 
does not limit the search for extra-terrestrial life based on a fix set of criteria. Under the view that the kind of living things is not clear-cut, but rather fuzzy, experimentation with the life's hallmarks can go on without any essentialist or definitionalist requirement for a full instantiation of life in a program. So, studies of computer viruses as digital organisms showing an increase in diversity and complexity, simulations of reproduction and selection phenomena, models of evolving populations in simulated ecosystems, simulations of metabolism and developmental processes, models of collective behaviors as flocking, etc., (see for example Dawkins 1989; Lindenmayer and Prusinkiewicz 1989; Packard 1989; Bagley et al. 1992; Ray 1992) can and must be carried out without it implying such a prescription for completeness. ${ }^{7}$

Finally, and importantly, a $\mathrm{PCK}^{*}$ conception is compatible with gradualist theories of the origins of life. As it allows for vague boundaries even in present-day forms of life, it picks out the continuous and gradual character of the transition from the non-living to the living. This is something that any sort of essentialist view would inexorably fail to do, as it presupposes a disruptive and exclusive classificatory scheme into living and non-living. If the essentialist wishes to avoid confronting empirical evidence and theories, she has two options: either she shows that a disruptive classificatory system is suited to fit empirical evidence (unlikely to be achieved), or she gives up on the very possibility that living systems be a natural kind (in which case the burden of proof is on her). The possibility of transition-cases, grey cases and blurred boundaries, on the other hand, lie at the core of a cluster approach. PCK* is modest enough so as not to influence, without further justification, our hypotheses of the origins of life. Indeed, it allows this research program to develop with fair autonomy (whereas it would be inevitably constrained by a strict definition or essence of life).

Notably, these valuable aspects could be obtained from applying HPC theory and related views. However, we have seen that HPC and related views are somewhat restrictive and endorse strong commitments that can be resisted. Our view, while retaining the advantageous features of the HPC tradition and related views (as opposed to an essentialist approach), accounts for cluster kinds with fewer challengeable assumptions.

\section{Objections and replies}

Our cluster kind approach might still be objected. Here, we consider and address actual and possible objections to our account.

\subsection{The explanatory limitation objection}

The merits of the cluster notions are generally acknowledged, but for some there is a high price to pay for such merits. For instance, Mark Bedau (2014, see also 2007) recognizes that a property-cluster approach to living systems neatly explains the existence of borderline cases and why life comes in degrees. However, he also states

\footnotetext{
${ }^{7}$ But there is no consensus among artificial life engineers or philosophers of artificial life that this research field's creations (both hardware and software) are, or will ever be, literally alive - a question that leads to two opposing trends in artificial life: "strong" versus "soft" (see Bedau 2007; Bedau and Cleland 2010, 217-218).
} 
that: "The main weakness with the cluster conception is that explanations end too quickly. The view does not explain why certain properties in the cluster are significant and other properties are not (...). The cluster conception cannot explain why life is characterized by one cluster of properties rather than another. The dimensions of the cluster of properties associated with living organisms is simply accepted as a contingent empirical fact. Those who expect some further explanation for life's characteristic properties will find the cluster conception of life unsatisfying". (Bedau 2014, 18, emphasis added). We call this the "explanatory limitation" objection.

In our view, Bedau's objection conflates different questions that result in a misconception of what accounts of natural kinds should minimally provide us with or explain. He explicitly refers to two questions that a cluster approach cannot answer, namely:

(i) Why is a kind (any kind) characterized by certain properties rather than by others?

(ii) Why are some properties more significant (common) than others for a kind $K$ ?

The first question could hardly be given a meaningful interpretation, either from an essentialist or an anti-essentialist perspective. In the former case, a kind would be characterized by its essential properties. And once we've found the essence of a kind (suppose we do), it just makes no sense to ask further "why is that the essence of this kind?" By definition, it could not have been differently (otherwise, it would not really count as an essence). Either it makes no sense to even pose the question, or one must be pleased with circular answers ("because that's its essence"). From the anti-essentialist perspective, the fact that a kind is characterized by certain properties rather than by others, while not a matter of definition, could only be regarded either as a brute fact of the universe or as a matter of divine intention. It amounts to questioning why the speed of light is $299,792,458 \mathrm{~m}$ per second rather than a different one (Lange 2002, ch. 4.3). It is true that a cluster approach (either Boyd's HPC theory or our modified account) cannot offer an answer to this question, but we contend that this was never the relevant or pertinent question. We doubt a view on natural kinds of any sort would aim at answering question (i). The reason why Bedau fails to note this, we believe, is that he is conflating this abstruse question with the others, fairly interesting ones.

Question (ii) might raise more reasonable concerns. The essentialistic answer to the second question would involve the essential/accidental distinction: some properties are more significant because they are essential, while others are merely accidental or contingent -an answer that would be unsatisfying for many people. Bedau suggests that the cluster view (any cluster view) would not even be able to deal with such a pertinent question, and we agree. However, note that question (ii) can be formulated when we have already identified kinds!

Now, this brings us naturally to the question that, in our view, a cluster account of natural kinds (in general), is aimed at answering:

(iii) How do we distinguish natural kinds from non-natural kinds?

This is, we contend, the crucial question from the point of view of the philosophical problem of natural kinds. Whereas natural kind theories do not aim at answering (i) and (ii), both Boyd's HPC theory and our PCK* offer criteria to answer this third question. 
The differences lie precisely in those criteria (cf. section 3), not in what both views assume to be their proper ultimate aim.

Under the essentialist view, questions (ii) and (iii) tend to collapse or, at least, can be easily conflated: natural kinds are distinguished from non-natural kinds because objects belonging to a given natural kind share essential properties that are also their identification criteria (iii), and the significant properties of a kind are those that are essential to it (ii). Interestingly enough, they too seem to collapse in Bedau's objection. He suggests that the cluster approach is flawed because it fails to answer question (ii). Thus, questions (ii) and (iii) seem to collapse in Bedau's expectation that a cluster approach to natural kinds should, in order to succeed as such (i.e., in order to be able to account for (iii)), also answer question (ii).

Of course, we do not mean to deny that there must be an explanation for the fact that, among the multiple properties that characterize living things, some are more common than others (question ii). Our point is, rather, that this question cannot be properly addressed from the philosophical point of view of identifying natural kinds, where the problem is how to distinguish natural classifications from non-natural ones. In fact, question (ii) is independent of this latter issue and calls for biological theories, models, generalizations, and hypotheses (Sober 1992, 763). If we could expect the kind concept of living systems to answer questions such as "why do most living things reproduce?", "why do metabolizing entities also reproduce?", or "why do replicating entities usually undergo evolution?" by itself, then what should we need biological science for? Biology must explain the occurrence and co-occurrence of properties of living things, but this is too much to ask of a natural kind concept.

\subsection{The membership determination objection}

A second objection states that HPC theory (and, likely, any other cluster view) fails to offer solid criteria to fix kind membership. This is due to the fact that both the property family and the underlying homeostatic mechanisms are defined in an open-ended manner, so that both may in principle change (Reydon 2009). So, "HPC theory, then, can only account for kinds whose extensions have already been fixed independently by other means" (Reydon 2009: 729). Interestingly, Bedau finds that a similar situation characterizes the debate on life definitions when one criterion to defining life is favored over the rest: "Gánti's life criteria and other lists of life's hallmarks always reflect and express some preconceptions about life. This might seem to beg the question of what life is. Any nonarbitrary list of life's hallmarks was presumably constructed by someone using some criterion to rule examples in or out" (Bedau 2007, 458).

Notably, Boyd acknowledged that determining membership can be difficult in some cases, although he didn't see this as a serious problem for his HPC theory (he even called it a "necessary indeterminacy," see Boyd 1991: 141-142), nor does Slater in his more recent work. But it surely sounds like a major problem if one believes that criteria to fix membership are the main thing a theory of natural kinds must provide us with, as well as if one inherits the requirements self-imposed by an essentialist view of natural kinds. Fixing membership, from an essentialist point of view, is a very simple thing to do in principle: for any given object, one only needs to find out whether the essence of the kind at stake is instantiated by it, provided that said essence has been identified. Yet identifying an essence is the truly puzzling part, whereas determining membership for a 
given object is arguably the lesser problems. The demand for a criterion to fix membership once and for all might be an essentialist requirement, as essences are the condition of possibility for such an infallible criterion.

It is true that determining membership once and for all seems to be beyond possibility in the case of life, but rather than a pitfall in our view of natural kinds, this is the very complicated state of affairs we started with in this paper: no set of properties is necessary and sufficient for something to be a living being, as we have seen in Section 2. If the essentialist wants to prove her view to be better than cluster approaches, then her argument cannot be that our approach fails to offer a criterion to determine membership for good, as this would amount to claiming that our approach fails for not being essentialist -a road we refused to take right from the start.

Our approach is not aimed at providing an algorithmic procedure to decide, for any given object, whether or not it is alive, because it is not intended to foreground or privilege one or a few of the various hallmarks of life as essential. Rather, the approach aims at providing a general framework for identifying natural kinds as opposed to arbitrary or mind-dependent groups of things. We do not believe that determining membership in a clear-cut, "yes or no" manner is a requisite to achieving the former goal; if so, this would be everybody's sin but the essentialist's (in fact, Reydon's objection is originally aimed at HPC theory).

From our point of view, then, a hidden essentialist-like assumption underlies this second objection. One might believe (as we do) that the aim of any theory of natural kinds is not so much to be able to fix membership, but to be able to distinguish natural kinds from non-natural kinds in the first place. If the greatest aim of a theory of natural kinds (and the minimum we must expect it to provide for) is rather some criteria with which one can distinguish natural kinds from arbitrary groupings of things, and if we have convincingly made the case that this can be achieved with our modification of Boyd's theory, then we see no reason to insist further on the membership determination worry. Determining membership is to a great extent a scientific task and should not be left for philosophy alone to settle.

\subsection{The triviality objection}

A final possible objection is that, if Boyd's theory is undemanding as it stands (from the point of view of essentialism, at least), then dropping any further requirement, as in $\mathrm{PCK}^{*}$-view, would render the notion of natural kind, in general, and the kind concept of life, particularly, somewhat trivial. Thus, one might first object that if $\mathrm{PCK}^{*}$ picks out the case of life it is only because it picks out just about any group of things as a natural kind.

It is true we could think of many ordinary groupings of things that share a family of properties (that is, condition (i)). For example, the group of objects contained in my wardrobe. However, uninteresting cases like these are removed by requirement (iii): it is not the case that every such grouping features in a scientific discipline with a heuristic and epistemic role. Moreover, requirement (ii) imposes a restriction as well: not every group of things science talks about would display a significant degree of stability. PCK* is narrow enough to discard undesired cases and broad enough to allow for fuzzy boundaries of living things and the smooth transition from the non-living to the living our 
biological theories demands for. There appears to be some sort of middle ground between a trivial notion of natural kinds and an over restrictive one.

Secondly, one might have the worry that the PCK* life concept, in particular, picks out just about any object as a living thing. But this is not really an issue so likely to arise. It is not the case that our approach would simply allow taking a rock or a soccer ball to be a living thing. The fact that there are difficult cases does not mean that every case has to be difficult. Obviously, there is no incompatibility between allowing for fuzzy boundaries and acknowledging straightforward cases. This is a point that takes us back to the membership determination objection. Still, someone could consider that such permissiveness (as to fuzziness, grey cases, and smoothness) must be deemed unacceptable. Yet such a consideration would amount to antagonizing biological theories and evidence for a smooth, continuous transition to life. The burden of proof is on the side of the essentialist - she needs to show that there is no such smoothness or fuzziness in the living world. Taken to the extreme, the objection implies the belief that any classification system is trivial if it does not classify things in a "black or white" manner, and that, conversely, only one capable of doing so is a good classification. Deep down, such belief has the implication that only essentialist classifications are to be desired and aimed at. As far as we know, in some cases, the natural world does not display joints to be carved at: there is no telling where a certain bone ends and another begins.

\section{Final remarks}

Lists of properties have been productively used in various biological sciences despite the difficulties to find a proper definition of living things. What we have proposed here is to redirect the discussion shifting the focus of attention from the quest to necessary and sufficient conditions towards a philosophical framework that allows conceiving of living systems as picking out a natural kind that is not to be defined in those terms. We took a cluster approach that partly shares the spirit of the HPC tradition but also incorporates modifications that enable the achievement of the same goals with fewer assumptions and commitments. With this framework, we accounted for life as a natural kind. However, our proposal was not designed ad hoc, since the need for those modifications was argued for with fair independence from the requirements imposed by our case study. In fact, it is a matter of further work to show how this view can be generalized and applied to other cases. The resulting view is free from HPC's limitations and assumptions and is a bit more flexible without being extremely permissive. In order to come up with a naturalistic account, we focused on research in those particular fields that, as has been argued, seem to require a definition of life in order to be carried out. The PCK* account of living systems helps making sense of scientific practice and the fact that certain objects of biological inquiry, without being paradigmatic cases of living things, can be regarded as and approached as objects standing more or less close to the paradigmatic ones.

One might wonder what we gain from conceiving of life as a $\mathrm{PCK}^{*}$-kind, given the ways in which we have relaxed long-standing requirements and expectations present in the HPC tradition. ${ }^{8}$ It might be argued that HPC would tell us something interesting

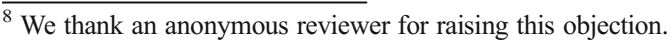


about the natural kind since it requires to identify the homeostatic mechanisms clustering its properties. This is true in the very general sense that causal mechanistic explanations of phenomena are considered to be interesting (with, however, the obvious warning that scientific explanation is not necessarily mechanistic). Yet we need to ask: did one need to have established that life was a HPC-kind as a condition of possibility of acquiring and valuing such causal explanations? In our view, one needs not. If such a causal explanation is interesting, it is so regardless of whether or not life is a HPC-kind. Moreover: regardless of whether or not life is a natural kind at all! Maybe we would need to rethink what HPC has to offer, as opposed to no natural kind view at all. Once again, expectations regarding philosophical theories of natural kinds could use a rethink. What we do aim to offer is probably less grandiloquent than others would expect from philosophical accounts of natural kinds, but we do not find such modesty impairing. What we gain from a PCK* view of life is a better understanding of cluster kinds in general and of life as a natural kind. "Better understanding" must be read in connection with the virtues of our approach developed in section 4, and also to our discussion of HPC's limitations and problems in section 3. In short, PCK* allows us to better understand of life as a natural kind with fewer commitments than the standard HPC tradition. Ultimately, a naturalistic approach to natural kinds also contribute to a scientifically informed philosophy of natural kinds.

Lastly, $\mathrm{PCK}^{*}$ is not meant to be an all-embracing view of natural kinds or that every kind in biology is a $\mathrm{PCK}^{*}$-kind. Rather, what we claim is that $\mathrm{PCK}^{*}$ is a more adequate and more naturalistic view of certain kinds characterized by non-perfect co-occurrence of properties and fuzzy boundaries, such as the case of living systems in general (specifically, more adequate and naturalistic than HPC). Natural kinds come in different varieties (Reydon 2009; Ereshefsky and Reydon 2015) and it has already been argued that it is unlikely that any attempts to come up with a general account of natural kinds will succeed (Ludwig 2018). As other philosophers do, we fail to see this as an unacceptable situation:

Arguably Hacking's argument against natural kinds is compelling because of a strong underlying presumption of the unity of science in some form or other in discussions of natural kinds. But if we don't accept this unity in the first place then the critique of natural kind concepts failing to apply uniformly to terms like "phosphorous" or "tiger" is misdirected. The job of a natural kind concept would never be to provide conceptual unity across disciplines but rather to provide a platform for exploring important and significant differences across an essentially disunified science (MacLeod and Reydon 2013, 96).

Acknowledgements Funding for this work was provided by grant GV/EJ IT590-13 from the Government of the Basque Country; grant FFI2014-52173-P from Ministerio de Economía y Competitividad (Spain) and FEDER funds from the EC; grant and FFI2013-47849-P from Ministerio de Economía y Competitividad (Spain); grant Olimpia Lombardi BID PICT 2014-2812 from Agencia Nacional de Promoción Científica y Tecnológica (Argentina); Consejo Nacional de Investigaciones Científicas y Tecnológicas (Argentina); and the Templeton Foundation Project "From Biological Practice to Scientific Metaphysics" (50191).

We thank Cristian López, Antonio Diéguez, Marc Ereshefsky, Marcel Weber and members of the Lake Geneva Biological Interest Group, and two anonymous reviewers for insightful comments on previous draft. 
Open Access This article is distributed under the terms of the Creative Commons Attribution 4.0 International License (http://creativecommons.org/licenses/by/4.0/), which permits unrestricted use, distribution, and reproduction in any medium, provided you give appropriate credit to the original author(s) and the source, provide a link to the Creative Commons license, and indicate if changes were made.

\section{References}

Alberts, B., Johnson, A., Lewis, J., Raff, M., Roberts, K., \& Walter, P. (2002). Molecular biology of the cell (4th ed.). New York: Garland Science.

Bagley, R., Farmer, J., \& Fontana, W. (1992). Evolution of a metabolism. In C. Langton, C. Taylor, J. Farmer, \& S. Rasmunssen (Eds.), Artificial Life II (pp. 141-158). Reading: Addison-Wesley.

Bedau, M. (2007). What is life? In S. Sarkar \& A. Plutynski (Eds.), A companion to the philosophy of biology (pp. 455-471). New York: Blackwell.

Bedau, M. (2014). The nature of life. In S. Luper (Ed.), The Cambridge companion to life and death (pp. 1329). Cambridge: Cambridge University Press.

Bedau, M., \& Cleland, C. (Eds.). (2010). The nature of life. Classical and contemporary perspectives philosophy and science. Cambridge: Cambridge University Press.

Benner, S. (2010). Defining Life. Astrobiology, 10(10), 1021-1030.

Bird, A., \& Tobin, E. (2015). "Natural Kinds." In E. Zalta (Ed.), The Stanford Encyclopedia of Philosophy (Spring 2015 Edition). URL = http://plato.stanford.edu/archives/spr2015/entries/natural-kinds/

Boyd, R. (1991). Realism, anti-foundationalism, and the enthusiasm for natural kinds. Philosophical Studies, $61,127-148$.

Boyd, R. (1999). Homeostasis, species and higher taxa. In R. Wilson (Ed.), Species: New interdisciplinary essays (pp. 141-185). The MIT Press: Cambridge.

Brussow, H. (2009). The not so universal tree of life or the place of viruses in the living world. Philosophical Transactions of the Royal Society of London B, Biological Sciences, 364, 2263-2274.

Campisi, E., Cardone, F., Graziano, S., Galeno, R., \& Pocchiari, M. (2015). Proteomics applications in prion biology and structure. Expert Review of Proteomics, 9(6), 649-666.

Claverie, J. M., Ogata, H., Audic, S., Arbergel, C., Suhre, K., \& Fournier, P. E. (2006). Mimivirus and the emerging concept of "giant" virus. Virus Research, 117(1), 133-144.

Cleland, C. (2012). Life without definitions. Synthese, 185, 125-144.

Cleland, C., \& Chyba, C. (2002). Defining 'Life'. Origins of Life and Evolution of the Biosphere, 32, 387-393.

Dawkins, R. (1989). The evolution of Evolvability. In C. Langton (Ed.), Artificial Life (pp. 201-220). Reading: Addison-Wesley.

Diéguez, A. (2013). Life as a homeostatic property cluster. Biological Theory, 7(2), 180-186.

Dupré, J. (1993). The disorder of things. Metaphysical foundations of the disunity of science. Cambridge: Harvard University Press.

Dupré, J., \& O’Malley, M. (2009). Varieties of living things: Life at the intersection of lineage and metabolism. Philosophy and Theory in Biology, 1, 1-25. https://doi.org/10.3998/ptb.6959004.0001.003.

Dyson, F. (1985). Origins of life. Cambridge: Cambridge University Press.

Emmeche, C. (1994). Is life a multiverse phenomenon? In C. Langton (Ed.), Artificial Life III (pp. 553-568). Reading: Addison-Wesley.

Ereshefsky, M., \& Reydon, T. (2015). Scientific kinds. Philosophical Studies, 172, 969-986.

Farmer, J., \& Belin, A. (1992). “Artificial life: The coming evolution.” In C. Langton et al. (Eds.), Artificial life $1 \mathrm{I}(815-40)$.

Forterre, P. (2010). Defining life: The virus viewpoint. Origins of Life and Evolution of the Biosphere, 40, 151-160.

Forterre, P., Krupovic, M., \& Prangishvili, D. (2014). Cellular domains and viral lineages. Trends in Microbiology, 22(10), 554-558.

Fox Keller, E. (2002). Making sense of life: Explaining biological development with models, metaphors, and machines. Cambridge: Harvard University Press.

Gánti, T. (2003). The principles of life. Oxford: Oxford University Press.

Gayon, J. (2010). Defining life: Synthesis and conclusions. Origins of Life and Evolution of Biospheres, 40, $231-244$.

Ghiselin, M. (1974). A radical solution to the species problem. Systematic Zoology, 23, 536-544.

Griffiths, P. (1999). Squaring the circle: Natural kinds with historical essences. In R. A. Wilson (Ed.), Species: New interdisciplinary essays (pp. 209-228). Cambridge: MIT Press.

Hacking, I. (1991). A tradition of natural kinds. Philosophical Studies, 61, 109-126. 
Häggqvist, S. (2005). Kinds, Projectibility and explanation. Croatian Journal of Philosophy, 5(13), 71-87.

Häring, M., Vestergaard, G., Rachel, R., Chen, L., Garrett, R. A., \& Prangishvili, D. (2005). Virology: Independent virus development outside a host. Nature, 436, 1101-1102.

Hull, D. (1978). A matter of individuality. Philosophy of Science, 45, 335-360.

Kondepudi, D., Kaufman, R., \& Singh, N. (1990). Chiral symmetry breaking in sodium chlorate crystallization. Science, 250, 975-976.

Kornblith, H. (1993). Inductive inference and its natural ground. Cambridge: MIT Press.

Koshland, D. (2002). The seven pillars of life. Science, 295, 2215-2216.

Kripke, S. (1980). Naming and necessity. Cambridge: Harvard University Press.

Lange, M. (1996). Life, "artificial life" and scientific explanation. Philosophy of Science, 63(2), 225-244.

Lange, M. (2000). Natural Laws in scientific practice. New York: Oxford University Press.

Lange, M. (2002). An introduction to philosophy of physics: Locality, fields, energy and mass. Oxford: Blackwell Publishing.

Langton, C. (1989). Artificial life. In C. Langton (Ed.), Artificial Life (pp. 1-47). Reading: Addison- Wesley.

Lederberg, J. (1960). Exobiology: Approaches to life beyond the earth. Science, 132, 393-400.

Li, J., Browning, S., Mahal, S., Oelschlegel, A., \& Weissmann, C. (2010). Darwinian evolution of prions in cell structure. Science, 327, 869-872.

Lindenmayer, A., \& Prusinkiewicz, P. (1989). Developmental models of multicellular organisms: A computer graphics perspective. In C. Langton (Ed.), Artificial Life (pp. 221-249). Reading: Addison-Wesley.

Ludwig, D. (2018). Letting go of "natural kind". Towards a multidimensional framework of non-arbitrary classification. Philosophy of Science, 85(1), 31-52.

Luisi, P. (1998). About various definitions of life. Origins of Life and Evolution of the Biosphere, 28, 613-622.

Lwoff, A. (1957). The concept of virus. Journal of General Microbiology, 17(1), 239-253.

Machery, E. (2012). Why I stopped worrying about the definition of life... and why you should as well. Synthese, 185, 145-164.

MacLeod, M. (2013). Limitations of natural kind talk in the life sciences: Homology and other cases. Biological Theory, 7, 109-120.

MacLeod, M., \& Reydon, T. (2013). Natural kinds in philosophy and in the life sciences: Scholastic twilight or new Dawn? Biological Theory, 7(1), 89-99.

Malaterre, C. (2009). Can synthetic biology shed light on the origins of life? Biological Theory, 4(4), 357-367.

Manrubia, S. (2012). Modelling viral evolution and adaptation: Challenges and rewards. Current Opinion in Virology, 2, 531-537.

Mayr, E. (1966). Animal species and evolution. Boston: Harvard University Press.

Mayr, E. (1976). Evolution and the diversity of life. Cambridge: Harvard University Press.

Mayr, E. (1982). The growth of the biological thought. Cambridge: Harvard University Press.

Mayr, E. (1997). This is biology: The science of the living world. Cambridge: Belknap Press of Harvard University Press.

Millikan, R. (1999). Historical kinds and the special sciences. Philosophical Studies, 95, 45-65.

Millikan, R. (2000). On clear and confused ideas. Cambridge: Cambridge University Press.

Moreira, D., \& López-García, P. (2009). Ten reasons to exclude viruses from the tree of life. Nature Reviews Microbiology, 7, 306-311.

Needham, P. (2000). What is water? Analysis, 60, 13-21.

Needham, P. (2011). Microessentialism: What is the argument? Noûs, 45, 1-21.

Packard, N. (1989). Evolving bugs in a simulated ecosystem. In C. Langton (Ed.), Artificial Life (pp. 141155). Reading: Addison-Wesley.

Palacci, J., Sacanna, S., Steinberg, A. P., Pine, D., \& Chaikin, P. (2013). Living crystals of light-activated colloidal surfers. Science, 31 .

Pearson, H. (2008). 'Virophage' suggests viruses are alive. Nature, 454, 677.

Pennock, R. (2012). Negotiating boundaries in the definition of life: Wittgensteinian and Darwinian insights on resolving conceptual border conflicts. Synthese, 185(1), 5-23.

Putnam, H. (1973). Meaning and reference. Journal of Philosophy, 70, 699-711.

Ray, T. (1992). An approach to the synthesis of life. In C. Langton, C. Taylor, J. Farmer, \& S. Rasmunssen (Eds.), Artificial Life II (pp. 371-408). Reading: Addison-Wesley.

Reydon, T. (2009). How to fix membership: A problem for HPC theory and a solution. Philosophy of Science, 72 , $724-736$.

Ruiz-Mirazo, K., Peretó, J., \& Moreno, A. (2010). Defining life or bringing biology to life. Origins of Life and Evolution of the Biosphere, 40, 203-213. 
Ruthenberg, K. (2012). What is water? Some philosophical considerations. In H. P. Hahn, K. Cless, \& J. Soentgen (Eds.), People at the Well: Kinds, Usages and Meanings of Water in a Global Perspective (pp. 65-78). Frankfurt: Campus Verlag.

Schrödinger, E. (1944). What is life? The physical aspect of the living cell. Cambridge: Cambridge University Press.

Schulman, R., \& Winfree, E. (2007). Synthesis of crystals with a programmable kinetic barrier to nucleation. Proceedings of the National Academy of Sciences of the United States of America, 104(39), 15236-15241.

Slater, M. (2013). Cell types as natural kinds. Biological Theory, 7, 170-179.

Slater, M. (2015). Natural kindness. British Journal for the Philosophy of Science, 66, 375-411.

Sober, E. (1980). Evolution, population thinking and essentialism. Philosophy of Science, 47, 350-383.

Sober, E. (1992). Learning from functionalism. Prospects for strong artificial life. In C. Langton, C. Taylor, D. Farmer, \& S. Rasmussen (Eds.), Artificial life II (pp. 749-765). Massachusetts: Addison-Wesley.

Umerez, J. (1995). Semantic closure: A guiding notion to ground artificial life. In F. Morán, A. Moreno, J. Merelo, \& P. Chacón (Eds.), Advances in artificial life (pp. 77-94). Berlin: Springer Verlag.

van Brakel, J. (2005). On the inventors of XYZ. Foundations of Chemistry, 7, 57-84.

Van Regenmortel, M. (2007). Virus species and virus identification: Past and current controversies. Infection, Genetics and Evolution, 7, 133-144.

Wilson, R. (2005). Genes and the agents of life: the individual in the fragile sciences: biology. Cambridge: Cambridge University Press.

Wilson, R., Barker, M., \& Brigandt, I. (2007). When traditional essentialism fails: Biological natural kinds. Philosophical Topics, 35(1-2), 189-215. 